\title{
AVALIAÇÕES FENOTÍPICAS DA RAÇA BOVINA CURRALEIRO PÉ-DURO DO SEMIÁRIDO DO BRASIL
}

\author{
PHENOTYPIC EVALUATION OF CURRALEIRO PÉ-DURO BREED OF CATTLE FROM \\ SEMIARID AREAS OFBRAZIL
}

\author{
Carvalho, G.M.C. ${ }^{1 *}$; Fé da Silva, L.R. ${ }^{2}$; Almeida, M.J.O. ${ }^{1}$; Lima Neto, A.F. ${ }^{1}$ e Beffa, L.M. ${ }^{3}$
}

${ }^{1}$ Embrapa Meio-Norte. Teresina, PI. Brasil. *geraldo.carvalho@embrapa.br

2Universidade Federal do Piauí (UFPI). Teresina, PI. Brasil.

${ }^{3}$ Consultor IICA/Agrofuturo. Embrapa Meio-Norte. Brasil. mariobeffa@gmail.com

\section{PalaVRas ChaVE ADICIONAIS}

Bos taurus adaptado aos trópicos. Raças crioulas. Recursos genéticos animais.

\section{RESUMO}

O objetivo do presente trabalho foi avaliar algumas características qualitativas e quantitativas relacionadas com a pelagem, morfometria, desenvolvimento ponderal, carcaça e carne de bovinos Curraleiro Pé-Duro (CPD, Bos taurus taurus). Os dados analisados foram coletados nos animais do núcleo de conservação in situ da Empresa Brasileira de Pesquisa Agropecuária (EMBRAPA) localizado na unidade experimental no Estado do Piauí, Brasil. Os dados foram analisados pelo método dos quadrados mínimos (Proc GLM) contidos no pacote estatístico SAS. Os modelos variaram conforme as análises, assim como o nível de significância dos efeitos ambientais incluídos e de suas interações. Os pesos médios, entre machos e fêmeas, para peso ao nascer (PN), desmama (P210), ano (P365), 18 meses (P18m), 24 meses (P24m) e na idade adulta (PA) foram 21 e 19; 69 e 64; 99 e 93; 137 e 127; 168 e 152; 285 e $210 \mathrm{~kg}$, respectivamente. O rebanho apresentou variações de pelagens com predominância da castanho-avermelhada seguida da amarelada, com extremidades escuras nos membros anteriores, principalmente nos animais adultos. As medidas morfométricas, as avaliações de carcaça e carne apresentaram grande amplitude de resultados indicando ausência de seleção e melhoramento no rebanho avaliado. Devido à introdução de zebuínos (Bos indicus) em meados do século $X X$ e aos cruzamentos desordenados que se seguiram. $O$ gado bovino CPD representa

\section{AdDitiOnAL KEYWORDS}

Animal genetic resources. Creole breeds. Tropically adapted Bos taurus.

uma das raças brasileiras em risco de extinção, necessitando de estudos mais aprofundados que permitam sua conservação e uso sustentável em regiões tropicais.

\section{SUMMARY}

The purpose of this research was to evaluate some qualitative and quantitative traits related to coat color, morphometric measurements, growth performance, weight daily gain, carcass and meat of Curraleiro Pé-Duro cattle (Bos taurus taurus, CPD). Data were obtained from the in situ conservation nucleus which belongs to the Brazilian Agricultural Research Corporation (EMBRAPA), located in the experimental farm in Piauí State, Brazil. The records were analyzed by least square method (Proc GLM) enclosed in SAS statistical package. Used models varied according to the analysis as well as the significance levels of environmental effects and their interactions. Growth performance means, among males and females, for birth (PN), weaning (P210), yearling (P365), 18 months (P18m), 24 months (P24m) and mature weight (PA) were 21 and 19;69 and 64, 99 and 93,137 and 127, 168 and 152, 285 and $210 \mathrm{~kg}$, respectively. The herd presented variations in coat colors with a predominance of brown-red followed by yellow, been darker in front body extremities, especially in mature animals. Morphometric measurements, evaluations of 
carcass and meat analyzes exhibited a great range of results indicating the absence of selection and genetic improvement in the evaluated herd. Due to the introduction of zebu cattle (Bos indicus) in the mid-twentieth century and the disordered crossbreeding's that followed, the CPD are at risk of extinction, requiring further studies that will allow its conservation and sustainable use in tropical regions.

\section{INTRODUÇÃO}

Os animais domésticos existentes no Brasil tiveram sua origem na península Ibérica, foram introduzidos pelos colonizadores portugueses no início do século XVI. Segundo Mariante e Cavalcante (2006) quase todas as raças crioulas locais brasileiras tiveram como ancestrais bovinos portugueses e segundo Athanassof (1958) a raça Curraleiro Pé-Duro (CPD) originou-se a partir do tronco étnico Bos taurus ibericus. Dada a sua origem européia, é presumido pertencer à subespécie Bos taurus taurus (Britto, 1998; Issa et al., 2009). Segundo Primo (1992) os animais introduzidos em Pernambuco e Bahia eram originários da Ilha da Madeira e Cabo Verde, respectivamente, e poderiam ter sido portadores de genes zebuínos (Bos indicus) africanos ou ainda tê-los recebido após a chegada ao continente americano. Dentro dessa premissa, aliados à deriva genética e a pressão de seleção natural exercida pelo ambiente, o gado CPD se formou originando animais adaptados aos biomas onde foram submetidos. Segundo Azevedo et al. (2008) os bovinos dessa raça são bem adaptados ao trópico quente e seco não mudando suas medidas fisiológicas no decorrer do dia e do ano, apesar das altas temperaturas do ambiente onde são criados.

Domingues et al. (1956) sugeriram padrão racial provisório para a raça CPD indicando que esse padrão poderia ser modificado de acordo com as necessidades ambientais. Esses autores recomendaram que os CPD fossem selecionados tendo em vista sua resistência e adaptação ao meio e propuseram alturas e pesos mínimos, para machos e fêmeas, respectivamente, de 1,24 e $1,38 \mathrm{~m}$ e 380 e $300 \mathrm{~kg}$. Athanassof (1958) afirma que o gado crioulo dos sertões de Pernambuco e da Paraíba é o mesmo gado Curraleiro de Goiás e também sugere um padrão racial para esses bovinos cuja pelagem predominante é alaranjada sendo mais clara na região perineal e ventral, a linha dorso lobar mais aberta que o resto do pelame e com extremidades mais escuras na frente, aberturas naturais pretas, pele de grossura média, elástica e pelos luzidios. Conforme o autor acima citado a altura média na cernelha seria de 1,20 e 1,35 m e o peso adulto de 350 e $400 \mathrm{~kg}$ para vacas e touros, respectivamente. Segundo Primo (1992) o tamanho do CPD é reduzido, apresentando altura na cernelha de $1,10 \mathrm{~m}$ e peso vivo entre 250 e $300 \mathrm{~kg}$ na idade adulta e a pelagem apresenta grande variedade de cores sendo as mais frequentes a amarelada e avermelhada em suas diversas tonalidades com extremidades escuras nos membros anteriores do corpo.

Entretanto, antes de se conhecer o real potencial das raças crioulas, a necessidade de raças bovinas mais produtivas para o agronegócio da carne no Brasil levou à impotação de raças exóticas, principalmente zebuínos, a partir da metade do século XX. Cruzamentos absorventes de maneira desordenada levaram á substituição e erosão das raças locais, que embora bem adaptadas exibiam níveis inferiores de produtividades (Egito et al., 2002). Os criadores acreditavam que o melhoramento dos bovinos resultantes dos cruzamentos entre animais crioulos com os exóticos se dava pelo material genético exógeno, o que acabou por subjugar o potencial genético das raças nativas que foram consideradas inferiores pela maioria dos criadores (Camargo, 1990). Com o objetivo de se evitar a completa substituição dos bovinos CPD, a Empresa Brasileira de Pesquisa Agropecuária (EMBRAPA) iniciou um projeto para conservação in situ no Centro Nacional de 


\section{CARACTERIZAÇÃO FENOTÍPICA DOS BOVINOS CURRALEIRO PÉ-DURO}

Pesquisa Agropecuária do Meio-Norte (Embrapa Meio-Norte) localizado no Estado do Piauí em 1982.

Segundo Fitzhugh e Strauss (1992) elementos importantes nos programas nacionais de conservação incluem o inventário, a caracterização e a documentação dos dados obtidos. Em termos de pesquisa, as prioridades devem ser dadas à caracterização e avaliação das populações locais e a mensuração das diferenças entre e dentro das populações. Além disso, o crescimento após a desmama, as avaliações de carcaça e a qualidade da carne são essenciais para estabelecer o potencial de um germoplasma como alternativa genética para ser usado pela indústria de carne bovina (Casas e Cundiff, 2006). Diversos trabalhos foram realizados com o objetivo de se caracterizar morfometricamente algumas raças crioulas brasileiras (Domingues et al., 1956; Athanassof, 1958; Trovo e Primo, 1984; Mazza et al., 1992; Primo, 1992; Abreu et al., 2002; Bianchini et al., 2006; Pereira et al., 2008).

Algumas pesquisas têm sido conduzidas para se avaliar o potencial de raças taurinas tropicalmente adaptadas para serem utilizadas em cruzamentos com zebuínos e taurinos visando mater a adaptabilidade em ambientes mais quentes, mas mantendo a qualidade da carne (Cundiff e Gregory, 1999; Dikeman et al., 2005; Chase et al., 2005; Wheeler et al., 2010). Segundo MacManus et al. (2008), caso se confirme as previsões de aquecimento global, o interesse e procura por raças adaptadas será incrementado e levará a uma reavaliação do valor das raças locais e cruzamentos estratégicos poderão ocorrer. No entanto, os resultados de diferentes situações e modelos implicam em diferentes espécies ou disponibilidade de raças adaptadas. Em qualquer caso, podese esperar que apenas os germoplasmas bem caracterizados sejam utilizados visando elevar a adaptabilidade de raças de alto rendimento (FAO, 2009). Assim sendo, o objetivo do presente trabalho foi avaliar a morfometria, o desenvolvimento ponderal, as características de carcaça e a qualidade da carne dos bovinos CPD para ser uma opção em programas de melhoramento genético e ainda garantir a conservação e uso sustentado desse germoplasma singular.

\section{MATERIALE MÉTODOS}

Os dados analizados na presente pesquisa foram coletados entre 2007 e 2010 no rebanho de conservação in situ da Embrapa Meio-Norte, localizado no Estado do Piauí $\left(8^{\circ} 22^{\prime} \mathrm{S} \mathrm{e} 42^{\circ} 15^{\prime} \mathrm{O}\right)$, constituído de aproximadamente 300 animais. O campo experimental tem altitude média de $222 \mathrm{~m}$, com clima típico BSh segundo classificação de Köppen, apresentando temperatura mínima de $21{ }^{\circ} \mathrm{C}$ e máxima de $41^{\circ} \mathrm{C}$ absolutas, com média anual de $29^{\circ} \mathrm{C}$. A umidade relativa do ar é de $53 \%$ em média, com precipitação pluviométrica de $600 \mathrm{~mm}$ anuais com distribuição irregular, apresentando estação seca e chuvosa com duração de 9 e 3 meses, respectivamente. A proximidade do equador faz com que a luminosidade seja muito intensa apresentando $21 \mathrm{MJ} / \mathrm{m}^{2}$ de radiação solar e a velocidade média do vento é de 7 $\mathrm{m} / \mathrm{s}$. Os dados metereológicos são medidos diuturnamente na estação existente no próprio campo experimental. A fazenda onde o rebanho é criado possui 1485 hectares com predomínio de terras de chapadas, sendo maior a ocorrência de latossolos amarelos de textura média. Em áreas de encostas e desníveis, ocorrem solos concrecionários e solos litólicos, com pedregosidade na superfície. As chapadas e encostas são cobertas por vegetação de caatinga hipoxerófila com crescimento sazonal, constituindo a fonte predominante de nutrição dos bovinos, sendo naturalmente enriquecida com folhas e vágens de leguminosas arbóreas e arbustos (Carvalho, 1984). A suplementação adicional é de água fresca em tanques e sal mineral em cochos cobertos.

Os dados relativos ao desenvolvimento 
ponderal foram coletados de animais nascidos nos anos de 2008, 2009 e 2010 em dois períodos de nascimento, sendo o primeiro período (P1) constituído pelo grupo de animais nascidos nos meses de janeiro, fevereiro e março e o segundo período (P2) o grupo de bezerros nascidos nos meses de abril, maio e junho, nos anos avaliados. Até a desmama as crias permaneceram em tempo integral junto ás mães quando foram desmamados em uma única data no final de setembro e então separados por sexo e encaminhado a pastagens distintas. As variáveis analisadas foram peso ao nascimento (PN); ao desmame (P210); ao ano (P365); aos 18 meses (P18m) e aos 24 meses (P24m). Também foi avaliado o ganho de peso diário entre o PN e P210 (GPM210); entre P210 e P365 (GPM365); entre P365 e P18m (GPM18m); entre o P18m e P24m (GPM24m) e entre o PN e P24m o ganho diário total (GPMtotal). Para PN, P210, P365, GPM210, GPM365 foram avaliados 100 animais, sendo 50 machos e 50 fêmeas; já em P18m, P24m, GPM18m, GPM24m e GPM total foram avaliados 74 animais, sendo 37 machos e 37 fêmeas, uma vez que as pesquisas ainda estão em andamento.

Para cálculo do peso adulto (PA) foram utilizados os dados relativos às pesagens dos anos de 2008, 2009 e 2010 de animais acima de 2 anos de idade. Os anos foram dividos em dois períodos para pesagem, sendo o período 1 (PA1) a pesagem realizada no fim da estação chuvosa (abril) e o período 2 (PA2) a pesagem realizada no final da estação seca (Novembro). O rebanho, por sua vez, foi divido em quatro grupos de idades (GI) para estudo mais detalhado do peso maduro e das medidas de exterior. Os animais com dois, três e quatro anos de idade constituiram o grupo de idade 1 (GI1); os animais entre cinco, seis e sete o grupo 2 (GI2); os com idade entre oito, nove e dez o grupo 3 (GI3) e os animais acima de onze anos o (GI4). As medidas morfométricas tomadas foram o comprimento da orelha (CO); largura da orelha (LO); largura da fronte (LF); altura da cabeça (AK); largura do espelho nasal (LE); altura do espelho (AE); comprimento do chifre (CCh); comprimento do corpo (CC) composta pelo dorso e garupa; altura da cernelha (AC); altura da garupa (AG); largura da garupa (LG); circunferência torácica (CT) e circunferência escrotal (CE).

Os dados coletados foram analizados pelo método dos quadrados mínimos através dos procedimentos gerais de modelos lineares (Proc GLM) contidos no pacote estatístico SAS (1995). Para o desempenho do crescimento o modelo utilizado incluiu os efeitos ambientais de sexo, período de nascimento, ano de nascimento e interação entre período de nascimento e ano; para P210, P365, P18m, P24m e GPM foi incluído nos modelos como covariável a idade do animal. Para medidas de exterior o modelo utilizado incluiu os efeitos de sexo, peso e idade como covariável. Já o dados relativos a carcaça e carne foram analisados em um modelo que incluiu os efeitos do PV ao abate e idade dos tourinhos como covariável. Para PA e medidas morfométricas dos adultos foi usado um modelo que incluiu o GI e o sexo. Nas análises estatísticas finais as respectivas médias foram comparadas pelo teste de Tukey (5\%) para estimar as significâncias das diferenças entre as mesmas. A descrição fenotípica de pelagem do rebanho foi conseguida através de observações individuais, de ambos os sexos, em animais adultos. A coloraçã o da pelagem foi classificada em conformidade com padrões previamente estabelecidos.

Dez tourinhos nascidos em 2008, foram abatidos para avaliação das carcaças e da qualidade da carne. Nessa etapa, as variáveis coletadas foram o peso vivo ao abate (PV); circunferência torácica (CT); altura da garupa (AG); altura da cernelha (AC). Após o abate o peso da carcaça quente (CQ); áreade-olho-de-lombo (AOL) e rendimento de carcaça (RC). Os animais foram abatidos em frigorífico comercial fiscalizado pelo serviço de inspeção federal (SIF) do Ministério da 


\section{CARACTERIZAÇÃO FENOTÍPICA DOS BOVINOS CURRALEIRO PÉ-DURO}

Agricultura Pecuária e Abastecimento (MAPA) e foram observadas todos os princípios éticos recomendados à experimentação animal. $\mathrm{O}$ pH da carne foi verificado no músculo Longissimus dorsi, após 24 horas de acondicionamento em câmara fria, na região entre a $12^{\mathrm{a}}$ e $13^{\mathrm{a}}$ costela, utilizando medidor de $\mathrm{pH}$ Analion Mod. PM 602. Ao mesmo tempo, foram coletados amostras do músculo Longissimus dorsi resfriado, para posterior realização da avaliação de maciez, coloração da carne e capacidade de retenção de água (CRA) no laboratório de avaliação de qualidade de carnes da Embrapa Pecuária sudeste em São Carlos, SP . A coloração da carne foi avaliada na forma de leitura no colorímetro Minolta (Minolta Chroma Meter CR-300, Minolta Corp., Ramsey, NJ).

A maciez foi avaliada através da força de cisalhamento (FC), a partir de amostras do músculo Longissimus dorsi. As amostras foram assadas até atingirem temperatura interna de $71^{\circ} \mathrm{C}$, e após foram resfriadas até atingirem uma temperatura de $20^{\circ} \mathrm{C}$ foi feita a medição da força, através do equipamento Warner-Bratzler shear. A capacidade de retenção de água foi obtida por diferença entre os pesos de uma amostra de carne, de aproximadamente $2 \mathrm{~g}$, antes e depois de ser submetida à pressão de $10 \mathrm{~kg}$, durante 5 minutos. A perda por cocção foi determinada pela diferença entre os pesos do bife antes e depois do cozimento até a amostra atingir em seu interior $70^{\circ} \mathrm{C}$. Foi realizada análise de variância e teste de médias utilizando o programa SAS.

\section{RESULTADOSEDISCUSSÃO}

\section{DESEMPENHO PONDERAL}

A significância dos efeitos ambientais sobre o crescimento dos CPD do nascimento aos dois anos de idade e as respectivas médias são apresentadas nas tabelas I e II. A importância dos efeitos variou conforme a idade dos animais, mas em P18m e P24m os efeitos significativos foram o sexo e a interação período/ano de nascimento, sendo que esta última também influenciou no GPMtotal uma vez que os animais foram criados a pasto sem suplementação alimentar. O que seria um indicativo de que o fornecimento adicional de alimentos poderia melhorar o desempenho dos bezerros em relação ao crescimento. O mês (período) de nascimento influenciou o PN significativamente $(\mathrm{p}<0,01)$ e também na desmama $(\mathrm{p}<0,05)$ indicando que os animais que nasceram no $\mathrm{P} 2$ foram favorecidos pelo acesso às melhores pastagens por suas mães antes do nascimento; por outro lado os que nasceram no P1 tiveram acesso a esses melhores pastos e apresentaram maior peso á desmama do que os nascidos no $\mathrm{P} 2$. Entretanto o período de nascimento não influenciou o peso nas avaliações subsequentes. Já a covariável idade influenciou significativamente todas as pesagens, quando os animais mais novos foram os mais leves sendo um indicativo que uma mudança no manejo para concentrar os nascimentos no P1 elevaria o peso na desmama, ao ano, sobreano e em P24m com maior retorno financeiro ao criador. Pela análise das médias pelo teste de Tukey os produtos machos foram mais pesados que as fêmeas em todas as avaliações do crescimento, entretanto o GPM210, GPM363, GPM18m e GPD24m foi igual entre os sexos nesses períodos, tendo sido diferente em favor dos machos apenas quando foi avaliado todo o GPDtotal quando se incluiu a pré-desmama. Os animais nascidos no P1 também apresentaram diferenças significativas a seu favor em relação ao GPDtotal se comparado aos nascidos no P2.

Entre as raças crioulas bovinas do Brasil, a raça Caracu é a mais bem avaliada e se encontra inserida no mercado produtivo, não correndo mais risco de extinção. O Caracu é utilizado como raça pura para corte e leite e ainda indicado em cruzamentos visando-se a heterose e a melhoria da carne produzida. A raça Crioula Lageana, já reconhecida como tal, se encontra em pleno 
Tabela I. Resumo da análise de variância de alguns efeitos ambientais no desempenho dos Curraleiro Pé-Duro. (Variance analysis summary of environmental effects on Curraleiro Pé-Duro growth and daily gain average).

\begin{tabular}{lcccccccccc}
\hline & PN & P210 & P365 & P18 & P24 & GPM210 & GPM365 & GPM18 & GPM24 & GPMtotal \\
\hline Sexo & $* *$ & NS & NS & $*$ & $* *$ & NS & NS & $*$ & NS & $*$ \\
Período & $* *$ & NS & NS & NS & NS & $*$ & NS & NS & NS & NS \\
Ano & $*$ & $* *$ & $* *$ & NS & NS & $* *$ & $* *$ & NS & $*$ & NS \\
Período/ano & NS & $*$ & $* *$ & $* *$ & $*$ & $* *$ & NS & $* *$ & NS & $*$ \\
Idade em dias & - & $* *$ & $* *$ & $*$ & $* *$ & NS & $*$ & NS & NS & NS \\
\hline
\end{tabular}

NS: Não significante; ${ }^{*} \mathrm{p}<0,05 ;{ }^{* *} \mathrm{p}<0,01 ; \mathrm{PN}$ : peso ao nascer; $\mathrm{P} 210$ : peso à desmama; $\mathrm{P} 365$ : peso ao ano; P18: peso aos 18 meses; P24: peso aos 24 meses; GPM: ganho de peso diário, PN-P210, P210-P365, P365-P18 e P18-P24; GPMtotal: ganho de peso médio diário entre o PN-P24.

processo de avaliação de suas potencialidades, conta com uma Associação de Criadores ativa e a ampliação dos rebanhos e procura por animais é visível. Pereira et al. (2008) apresentado trabalho de 23 anos de seleção na raça Caracu para peso pósdesmama relatou PN e P210 de 32 e $204 \mathrm{~kg}$ respectivamente; $\mathrm{P} 365$ para machos de 334 $\mathrm{kg}$ e $\mathrm{P} 18 \mathrm{~m}$ para fêmeas de $281 \mathrm{~kg}$. A pressão de seleção contínua para elevar o peso dos machos em P365 e das fêmeas em P18m elevou o PA da população selecionada, o que não seria desejável em regiões como o semiárido. Abreu et al. (2002) avaliando as características fenotípicas de bovinos Pantaneiros relatou PN e P210 para machos e fêmeas, de 25 e $27 \mathrm{~kg}$ e 112 e $116 \mathrm{~kg}$, respectivamente. Os autores também relataram efeito significativo de mês de nascimento na desmama quando os bezerros nascidos no final da estação das chuvas apresentaram pesos médios $25 \mathrm{~kg}$ acima dos nascidos no final do perído seco. O período de nascimento na presente avaliação também influenciou o peso ao nascer e em épocas subsequentes.

Tabela II. Desempenho ponderal de Curraleiros, machos e fêmeas, do nascimento aos dois anos de idade e ganho de peso médio diário, em kg, entre as pesagens. (Curraleiro performance of males and females, from birth to two years of age and weight daily gain average between weights, in $\mathrm{kg}$ ).

\begin{tabular}{lcccccccccc}
\hline & PN & P210 & P365 & P18 & P24 & GPM210 & GPM365 & GPM18 & GPM24 & GPMtotal \\
\hline Machos & $21,30^{\mathrm{A}}$ & $68,86^{\mathrm{A}}$ & $98,51^{\mathrm{A}}$ & $137,27^{\mathrm{A}}$ & $168,05^{\mathrm{A}}$ & $0,215^{\mathrm{AA}}$ & $0,198 \mathrm{~A}$ & $0,230^{\mathrm{A}}$ & $0,161^{\mathrm{A}}$ & $0,197^{\mathrm{A}}$ \\
Fêmeas & $19,06^{\mathrm{B}}$ & $64,16^{\mathrm{B}}$ & $92,53^{\mathrm{B}}$ & $126,62^{\mathrm{B}}$ & $152,89^{\mathrm{B}}$ & $0,212^{\mathrm{A}}$ & $0,191 \mathrm{~A}$ & $0,196^{\mathrm{A}}$ & $0,133^{\mathrm{A}}$ & $0,182^{\mathrm{B}}$ \\
P1 & $19,74^{\mathrm{b}}$ & $72,34^{\mathrm{a}}$ & $99,95^{\mathrm{a}}$ & $138,15^{\mathrm{a}}$ & $165,98^{\mathrm{a}}$ & $0,227^{\mathrm{a}}$ & $0,186^{\mathrm{a}}$ & $0,214^{\mathrm{a}}$ & $0,144^{\mathrm{a}}$ & $0,194^{\mathrm{a}}$ \\
P2 & $21,58^{\mathrm{a}}$ & $47,54^{\mathrm{b}}$ & $81,17^{\mathrm{b}}$ & $107,53^{\mathrm{b}}$ & $138,81^{\mathrm{b}}$ & $0,170^{\mathrm{b}}$ & $0,220^{\mathrm{b}}$ & $0,210^{\mathrm{a}}$ & $0,160^{\mathrm{a}}$ & $0,174^{\mathrm{b}}$ \\
N & 100 & 100 & 100 & 74 & 74 & 100 & 100 & 74 & 74 & 74 \\
\hline
\end{tabular}

ABMédias com letras maiúsculas diferentes na mesma coluna são diferentes (Tukey); abMédias com letras minúsculas diferentes na mesma coluna são diferentes (Tukey); P1: período de nascimento entre janeiro e março; P2: período de nascimentoabril e junho; PN: peso ao nascer; P210: peso à desmama; P365: peso ao ano; P18: peso aos 18 meses; P24: peso aos 24 meses; GPM: ganho de peso diário, PN-P210, P210-P365, P365-P18 e P18-P24; GPMtotal: ganho de peso médio diário entre o PN-P24.

Archivos de zootecnia vol. 62, núm. 237, p. 14. 


\section{CARACTERIZAÇÃO FENOTÍPICA DOS BOVINOS CURRALEIRO PÉ-DURO}

\section{AVALIAÇÃO DE CARCAÇA E QUALIDADE DA CARNE}

A tabela III contém médias, desvio padrão, valores mínimo e máximo do peso vivo ao abate ao abate, algumas medidas de exterior, carcaça e qualidade da carne de tourinhos abatidos aos 28 meses de idade. Diferente da maioria das avaliações em teste de progênie, onde os animais são criados em sistemas intensivos, com dietas especiais e em ambientes distintos de onde geralmente são criados, esse experimento foi conduzido a campo em pastagens naturais com fornecimento de àgua e sal mineralizado. $\mathrm{O}$ objetivo principal dessa avaliação não foi o ganho de peso e sim ampliar os conhecimentos sobre essa raça e investigar o potencial e variablididade dos indivíduos dentro da raça. Os resultados obtidos nessa avaliação estão em conformidade com diversos autores que ao caracterizarem tipos biológicos distintos de incluindo o crioulo Romosinuano nos Estados Unidos, em relação à carcaça, produção de carne e palatabilidade constataram maior variabilidade dentro de raça do que entre raças (Cundiff e Gregory, 1999; Chase et al., 2005 e Wheeler et al., 2010). Dentre as características de interesse econômico, o PV, a CQ, AOL, RC e FC os valores encontrados no presente estudo variaram entre 186 e $298 \mathrm{~kg}$; 74 e $152 \mathrm{~kg} ; 37$ e $58 \mathrm{~cm}^{2} ; 39$ e $50 \% ; 2,9$ e 10,36 $\mathrm{kg}$ respectivamente, confirmando um campo vasto para o melhoramento genético nos bovinos curraleiro Pé-duro. Dikeman et al. (2005) avaliando carcaça e herdabilidade para qualidade da carne enunciou que valores de FC abaixo de $5.0 \mathrm{~kg}$ para maciez seria considerado como carne macia. No presente trabalho foi verificado valores mínimos de FC de até $2,9 \mathrm{~kg}$, sendo uma carne classificada em mercados exigentes como extremamente macia. Entretanto, a força de cisalhamento média foi 6,99 e a máxima de 10,36 , o que poderia ser em parte explicado pelo baixo acabamento da carcaça sem gordura de cobertura e marmoreio, uma vez que
Tabela III. Avaliação da morfometria, carcaça e carne de dez tourinhos Curraleiro Pé-Duro aos 28 meses. (Carcass, meat and morphometric evaluation of ten young Curraleiro bulls at 28 months of age).

\begin{tabular}{lcccc}
\hline \multicolumn{5}{c}{ Desvio } \\
& Média & Padrão & Mínimo Máximo \\
\hline Peso vivo (kg) & 226,21 & 33,67 & 186,00 & 298,00 \\
CT $(\mathrm{cm})$ & 146,60 & 8,41 & 135,00 & 159,00 \\
CE $(\mathrm{cm})$ & 25,00 & 2,00 & 21,00 & 28,00 \\
AG $(\mathrm{cm})$ & 112,80 & 3,65 & 107,00 & 119,00 \\
AC $(\mathrm{cm})$ & 107,00 & 5,29 & 101,00 & 117,00 \\
CQ $(\mathrm{kg})$ & 100,87 & 22,01 & 73,8 & 151,40 \\
AOL $\left(\mathrm{cm}^{2}\right)$ & 46,70 & 6,70 & 37,00 & 58,00 \\
RC $(\%)$ & 43,30 & 3,11 & 38,71 & 49,80 \\
FC (kg) & 6,99 & 2,29 & 2,97 & 10,36 \\
CRA (\%) & 78,42 & 1,82 & 74,24 & 81,24 \\
L* & 36,58 & 1,50 & 34,32 & 38,16 \\
a $^{*}$ & 13,05 & 0,80 & 11,49 & 14,47 \\
b* & 10,98 & 0,91 & 9,84 & 12,52 \\
PPC (\%) & 23,19 & 2,41 & 18,93 & 26,47 \\
pH & 5,86 & 0,24 & 5,60 & 6,32 \\
\hline
\end{tabular}

CT: circunferência torácica; $C E$ : circunferência escrotal; AG: altura na garupa; AC: altura da cernelha; $\mathrm{CQ}$ : carcaça quente; $\mathrm{AOL}$ : área de olhode-lombo; RC: rendimento de carcaça; FC: força de cisalhamento; CRA: capacidade de retenção de água; $L^{*}$, onde $L^{*}$ é o croma associado à luminosidade; $a^{*}$ : intensidade de vermelho de vermelho e $b^{*}$ : intensidade de amarelo na carne; PPC: perda por cocção.

foram criados a pasto em sistema de baixo input.

O valor médio de $\mathrm{pH}$ da carne observado foi de 5,86, variando de 5,6 a 6,32. Segundo Abularach et al., 1998, valores de $\mathrm{pH}$ final entre 5,40 e 5,60 são considerados normais para carne bovina. Considerando o trabalho acima citado, os valores encontrados no presente trabalho podem ser considerados atípicos e as carnes com pH acima 5,86 (média dessa pesquisa) pode resultar em carnes com corte escuro, retenção de água maior e vida de prateleira mais curta do que canres com $\mathrm{pH}$ inferior. Talvez o manejo inadequado dos animais no pré-abate tenha 
levado à redução do $\mathrm{pH}$ na carne e também o fato dos animais não serem castrados poderia ter elevado o estresse ante mortem e ainda sujeitos ao estresse hierárquico. A carne avaliada apresentou cor vermelha com lumonosidade ( $\mathrm{L}^{*}$ ) média entre 34,32 (mais escura) a 38,16 (mais clara) com média de 36,58 ; intensidade de vermelho $\left(\mathrm{a}^{*}\right)$ entre 11,49 e 14,47 com valor médio de 13,05 e a intensidade da cor amarela na carne verificado ficou entre 9,84 e 12,52 com média de 10,98 . A carne de touros jovens é normalmente mais escura que a de novilhos castrados e de novilhas (Purchas, 1990). Entretanto os resultados médios do presente trabalho de 36,58 mostram carnes mais escura que os resultados de publicados por Junqueira (1996) que foi de 38,08 em animais $1 / 2$ Nelore $1 / 2$ Marchigiana. Todavia os Curraleiros do presente trabalho apresentaram carne mais clara que os de tourinhos nelore relatados por Abularach et al. (1998) que foi de 34,85. A coloração da carne também é altamente influenciada pelos sistema de produção e procedimentos ante mortem. Segundo Shorthose (1989), pesquisa realizada em 3000 bovinos, terminados em pastagem ou confinamento, abatidos em diversos lotes, $70 \%$ dos lotes proveniente de confinamento apresentaram carne mais clara contera $16 \%$ dos lotes de pastagem. O outor atribuiu a diferença ao temperamento mais calmo dos animais de confinados. Segundo Silva et al. (2008), a intensidade da cor depende da concentração de pigmentos e do estado físico da carne, podendo sofrer variações devido a menor ou maior concentração de mioglobina e hemoglobina, dependendo do estado de sangria a qual os animais foram submetidos no abate.

Conforme Dikemam et al. (2005) a herdabilidade para maciez naquele estudo foi de 0,40 , para marmoreio 0,68 e para suculência de 0,37 , indicando que o melhoramento dentro de raça para essas variáveis qualitativas desejáveis podem ser alcançados. Segundo Felicio (1998), uma carne, que, além da qualidade óbvia tivesse cor, maciez, suculência e sabor assegurados, e que fosse apresentada ao consumidor précortada, corretamente embalada, com certificado de origem e indicações de preparo culinário, teria ao mesmo tempo qualidade óbvia e qualidade atrativa.

\section{AVALIAÇÃO MORFOMÉTRICA E DE PELAGENS}

A tabela IV mostra as mensurações detalhadas dos CPD no rebanho de conservação in situ da Embrapa Meio-Norte. Embora os machos apresentem diferenças significativas $(\mathrm{p}<0,05)$ em relação às fêmeas como na LF, CCh, AC, AG, CT e nos pesos adultos PA1 e PA2, em outras medidas parece não apresentar dimorfismo sexual significativo como no comprimento e largura da orelha, altura da cabeça, espelho nasal, comprimento do corpo e largura da garupa. Em relação aos grupos de idades, os animais do GI2 apresentaram CCh maior e LF por ser o grupo com maior número de touros uma vez que nessa faixa etária os touros já deixaram descendentes e tiveram sêmen coletado, sendo então disponibilizados para criadores em leilões anuais. O GI4 foi o grupo com maior número de vacas e apresentou diferença significativa na LG, justificando a ausência de partos distócicos no rebanho, como resultado de longos anos de seleção natural.

Os PA e AC mínimos indicados por Domingues et al. (1956) para machos e fêmeas, de 380 e $300 \mathrm{~kg}$; 124 e 138, respectivamente, foi usado como referência na escolha dos animais fundadores do rebanho in situ da Embrapa no Estado do Piauí (Carvalho e Pereira, 1981). Os resultados obtidos no presente estudo não conferem com o enunciado anterior uma vez que os PA e AC em média, para machos e fêmeas, foi de 284 e $210 \mathrm{~kg} ; 110$ e 107 , respectivamente. Por outro lado, Britto (1988) relatou resultado semelhante para AC de $110 \mathrm{~cm}$ para machos e $108 \mathrm{~cm}$ para fêmeas em um pequeno núcleo da Embrapa Cenargem, originários do rebanho de conservação in situ do Piauí 


\section{CARACTERIZAÇÃO FENOTÍPICA DOS BOVINOS CURRALEIRO PÉ-DURO}

Tabela IV. Medidas morfométricas de exterior $(\mathrm{cm})$ e peso adulto $(\mathrm{kg})$ de Curraleiro PéDuro, por grupos de idade e gênero. (Curraleiro Pé-Duro morphometric measurement's average $(\mathrm{cm})$ and mature weight $(\mathrm{kg})$ by age groups and gender).

\begin{tabular}{lccccccc}
\hline & $N$ & G1 & G2 & G3 & G4 & Macho & Fêmea \\
\hline CO & 143 & $15,96^{\mathrm{a}}$ & $15,91^{\mathrm{a}}$ & $15,77^{\mathrm{a}}$ & $15,33^{\mathrm{a}}$ & $16,17^{\mathrm{A}}$ & $15,71^{\mathrm{A}}$ \\
LO & 120 & $9,57^{\mathrm{a}}$ & $9,32^{\mathrm{a}}$ & $9,53^{\mathrm{a}}$ & $10,01^{\mathrm{a}}$ & $9,56^{\mathrm{A}}$ & $9,55^{\mathrm{A}}$ \\
LF & 120 & $17,22^{\mathrm{b}}$ & $18,39^{\mathrm{a}}$ & $18,11 \mathrm{~b}^{\mathrm{a}}$ & $17,93^{\mathrm{ba}}$ & $18,76^{\mathrm{A}}$ & $17,65^{\mathrm{B}}$ \\
AK & 120 & $40,35^{\mathrm{b}}$ & $42,29^{\mathrm{a}}$ & $42,11 \mathrm{~b}^{\mathrm{a}}$ & $42,81^{\mathrm{a}}$ & $42,44^{\mathrm{A}}$ & $41,52^{\mathrm{A}}$ \\
LE & 119 & $6,35^{\mathrm{a}}$ & $6,53^{\mathrm{a}}$ & $6,47^{\mathrm{a}}$ & $6,37^{\mathrm{a}}$ & $6,54^{\mathrm{A}}$ & $6,41^{\mathrm{A}}$ \\
AE & 119 & $6,78^{\mathrm{a}}$ & $7,16^{\mathrm{a}}$ & $7,36^{\mathrm{a}}$ & $7,27^{\mathrm{a}}$ & $7,14^{\mathrm{A}}$ & $7,04^{\mathrm{A}}$ \\
CCh & 117 & $39,89^{\mathrm{ba}}$ & $42,97^{\mathrm{a}}$ & $35,75^{\mathrm{b}}$ & $38,33 \mathrm{~b}^{\mathrm{a}}$ & $48,56^{\mathrm{A}}$ & $36,69^{\mathrm{B}}$ \\
CC & 290 & $108,82^{\mathrm{b}}$ & $117,51^{\mathrm{a}}$ & $118,75^{\mathrm{a}}$ & $120,38^{\mathrm{a}}$ & $115,51^{\mathrm{A}}$ & $114,44^{\mathrm{A}}$ \\
AC & 291 & $106,21^{\mathrm{a}}$ & $108,03^{\mathrm{a}}$ & $109,01^{\mathrm{a}}$ & $108,09^{\mathrm{a}}$ & $109,86^{\mathrm{A}}$ & $106,54^{\mathrm{B}}$ \\
AG & 220 & $107,65^{\mathrm{b}}$ & $111,36^{\mathrm{a}}$ & $112,92^{\mathrm{a}}$ & $112,14^{\mathrm{a}}$ & $111,49^{\mathrm{A}}$ & $109,95^{\mathrm{B}}$ \\
LG & 284 & $30,11^{\mathrm{b}}$ & $32,39^{\mathrm{b}}$ & $31,76^{\mathrm{b}}$ & $35,57^{\mathrm{a}}$ & $31,91^{\mathrm{A}}$ & $30,92^{\mathrm{A}}$ \\
CT & 291 & $135,48^{\mathrm{b}}$ & $144,17^{\mathrm{a}}$ & $145,95^{\mathrm{a}}$ & $146,29^{\mathrm{a}}$ & $144,97^{\mathrm{A}}$ & $139,82^{\mathrm{B}}$ \\
CE & 116 & $23,53^{\mathrm{b}}$ & $26,63^{\mathrm{ba}}$ & $28,64^{\mathrm{a}}$ & $30,01^{\mathrm{a}}$ & 27,21 & - \\
P1 & 99 & - & $256,68^{\mathrm{a}}$ & $249,56^{\mathrm{a}}$ & $236,47^{\mathrm{a}}$ & $296,22^{\mathrm{A}}$ & $222,45^{\mathrm{B}}$ \\
P2 & 99 & - & $236,12^{\mathrm{a}}$ & $224,94^{\mathrm{a}}$ & $204,93^{\mathrm{a}}$ & $273,70^{\mathrm{A}}$ & $197,13^{\mathrm{B}}$ \\
& & & & & & &
\end{tabular}

ABCMédias com letras maiúsculas iguais na mesma linha são iguais (Tukey); abcMédias com letras minúsculas iguais na mesma linha são iguais (Tukey).

(subamostra). Os resultados para AC e PA verificados no presente estudo estão também em concordância com os dados publicados por Primo (1992) de $110 \mathrm{~cm}$ e 285 $\mathrm{kg}$ para AC e PA. Bianchini et al. (2006), trabalhando com descendentes dos animais analizados por Trovo (1985), relataram para AC, CC e CT, em média para ambos os sexos medidas de 114, 131 e 167, respectivamente, estando acima dos resultados encontrados na presente análise, o que se justificaria devido a diferenças ambientais marcantes entre os locais das pesquisas, Caatinga e Cerrado.

Relativamente, quando comparado com raças comerciais taurinas e zebuínas, poucos trabalhos científicos foram realizados com a finalidade de se conhecer as características raciais dos bovinos crioulos do Brasil. Trabalhos antigos, realizados quando os bovinos Curraleiros ainda reinavam soberanos pelas terras tupiniquins são detalhistas apenas em relação à cor das pelagens mas pouco inferiram ou acrescentaram na avaliação zootécnica e produtiva dos rebanhos que acabaram sendo absorvidos pelos zebuínos e até mesmo por outras raças crioulas. Atualmente o Mocho Nacional foi incorporado ao Caracu e é registrado como tal e o mesmo deve ter acontecido com rebanhos Curraleiros que apresentavam peso adulto como os relatados por Athanassof (1958), $500 \mathrm{~kg}$ para machos e $380 \mathrm{~kg}$ para fêmeas.

As pelagens variam do vermelho ao preto sólidos, em suas diversas tonalidades e particularidades (óculos, ventre e ou dorso claro, rajado, cara branca (moura) e malhado. Todavia a maioria das pelagens, em $82 \%$ dos animais avaliados, foi alaranjada. A figura 1 mostra um touro e uma vaca com cria ao pé na pelagem característica da raça.

CORRELAÇÕES ENTRE AS VARIÁVEIS DE INTERESSE

As correlações fenotípicas entre medi- 


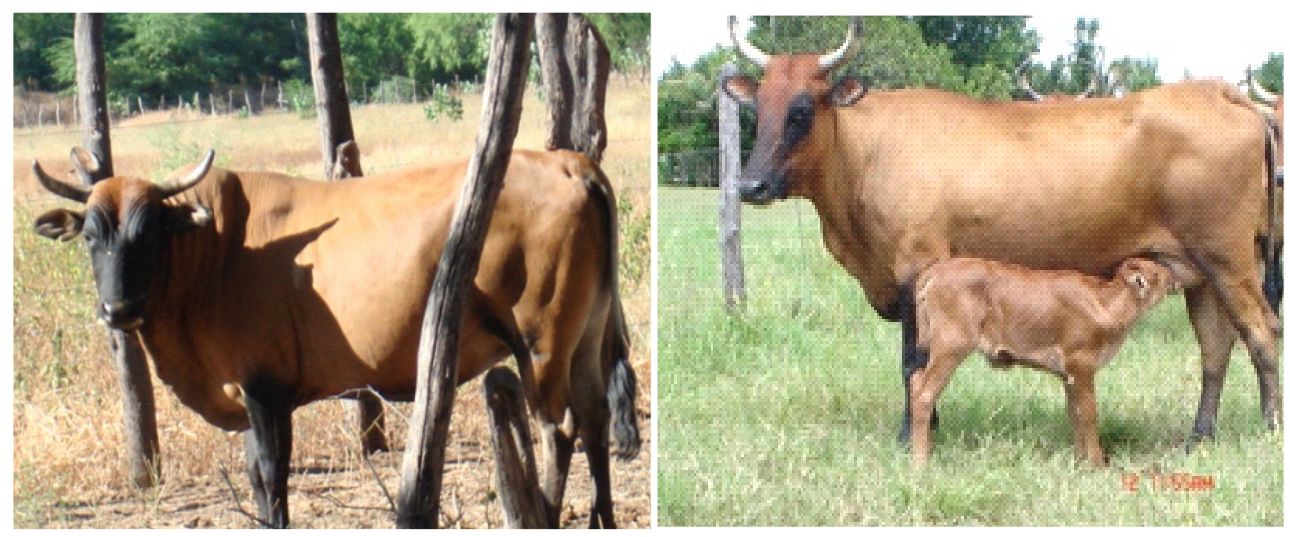

Figura 1. Touro e vaca com cria Curraleiro Pé-Duro. (Bull and cow with calf of Curraleiro PéDuro).

das morfométricas e peso vivo em diversas idades mostraram-se de moderadas a altas. Os resultados indicam que a seleção para peso em qualquer idade deve acarretar ganho de peso em pesagens posteriores e também aumento na estatura dos animais. Todavia os CPD apresentam-se bem adaptados ao ambiente em que vivem e o aumento de tamanho acarretaria em maior necessidade nutricional resultando em maiores inputs e despesas. Desta forma, para obtenção de animais com tamanho e peso adequados ao sistema de produção, faz-se necessário a utilização de um índice de seleção aliado a outras características de maior valor econômico como área de olho-de-lombo e rendimento de carcaça, restringindo o aumento de tamanho. Com o uso de varreduras ultrassonográficas esse índice poderia ser obtidos também em animais vivos, dividindo-se a área de olho-de-lombo pelo peso vivo e multiplicando-se por 100, que proporcionaria por sua vez a relação entre AOL por $100 \mathrm{~kg}$ de PV; ou de carcaça sabendo-se o rendimento da carcaça (Drake e Chapman, 2008). Esse procedimento poderia indentificar animais com bom ganho de peso em carne e não em ganho de peso também em medidas esqueléticas o que manteria o tamanho médio dos animais e promoveria o melhoramento das carcaças. Não obstante, a seleção em geral tem enfatizado o crescimento, o peso, o tamanho, favorecendo os animais com crescimento rápido, que leva ao aumento do peso adulto. O tamanho ideal está relacionado com requisitos nutricionais e produção de alimentos em cada ecossistema objetivando a eficiência de uma situação particular e o tamanho pequeno pode ser um benefício de acordo com o ambiente, segundo Cartwrigth e Blackburn (1989).

\section{CONCLUSÕES}

As pelagens do Curraleiro Pé-Duro variam do vermelho ao preto em suas diversas diluiçoes e particularidades. Todavia a maioria dos animais são de pelagem alaranjada.

Os animais dessa raça são de porte pequeno e bem adaptados ao ambiente onde sãos criados.

Mudar a estação de nascimento para o começo do año pode representar maior ganho de peso durante as fases de crescimento.

O uso de medidas de exterior e o ganho de peso como criterio de seleção poderá aumetar o tamanho médio da raça sem 


\section{CARACTERIZAÇÃO FENOTÍPICA DOS BOVINOS CURRALEIRO PÉ-DURO}

contudo melhorar o rendimento de carcaça e AOL. Melhor usar o índice como critério (peso vivo/AOL).

A grande amplitude nos resultados

\section{BIBLIOGRAFIA}

Abreu, U.G.P.; McManus, C.; Moreno-Bernal, F.E.; Lara, M.A.C. and Sereno, J.R.B. 2002. Genetic and environmental factors influencing birth and 205 day weights of Pantaneiro calves. Arch Zootec, 51: 83-89.

Abularach, M.L.S.; Rocha, C.E. e Felicio, P.E. 1998. Características de qualidade do contrafilé ( $m$. L. dorsi) de touros jovens da raça Nelore. Ciênc Tecnol Aliment, 18: 205-210.

Athanassof, N. 1958. Manual do criador de bovinos. $6^{a}$ ed. Melhoramentos. São Paulo. 818 pp.

Azevedo, D.M.R.R.; Alves, A.A. e Feitosa, F.S. 2008. Adaptabilidade de bovinos da raça PéDuro às condições climáticas do semiárido do Estado do Piauí. Arch Zootec, 57: 5-11.

Bianchini, E.; McManus, C.; Lucci, C.M.; Fernandes, M.C.B.; Prescott, E.; Mariante, A.S. e Egito, A.A. 2006. Características corporais associadas com a adaptação ao calor em bovinos naturalizados brasileiros. Pesqui Agropecu Bras, 41: 1413-1448.

Britto, C.M.C. 1988. Citogenética do Gado PéDuro. EDUFPI. Teresina. $80 \mathrm{pp}$.

Camargo, A.H.A. 1990. Ganado criollo del Brasil: Origen y características zootécnicas. Boletim de Informacion sobre los Recursos Geneticos Animales. FAO \& UNEP. Roma. pp. 11-16.

Cartwrigth, T.C. e Blackburn, H.D. 1989. Portability of animal breeding research to developing countries: beef cattle. J Anim Sci, 67: 342-347.

Carvalho, J.H. e Pereira P.R. 1981. Projeto para implantação de um núcleo de preservação de gado Pé-Duro ou Curraleiro. EMBRAPA/UEPAE. Teresina. 17 pp.

Carvalho, J.H. 1984. Relatório de atividades do núcleo de preservação do gado Pé-Duro ou Curraleiro. EMBRAPA/UEPAE. Teresina. $17 \mathrm{pp}$.

Casas, E. e Cundiff, L.V. 2006. Post-weaning growth and carcass traits in crossbred cattle from Hereford, Angus, Norwegian Red, Swedish Red and White, Friesian, and Wagyu maternal grandsires. J Anim Sci, 84: 305-310.

Chase, C.C.; Riley, D.G.; Olson, T.A. e Coleman, S. indicam um vasto campo para o melhoramento genético.

Mais pesquisas se faz necessário para se avaliar a raça em sua total potencialidade.

2005. Evaluation of Brahman and tropically adapted Bos taurus breeds in the humid subtropics. Research Report, USDA-ARS. http:/ /www.ars.usda.gov/research/projects/ projects.htm?ACCN_NO=405830(17/02/2011).

Cundiff, L.V. e Gregory, K.E. 1999. Searching for new genetic resources for composite populations. Rev Bras Reprod Anim, 23: 549-563.

Dikeman, M.E.; Pollack, E.J.; Zhang, Z.; Moser, D.W.; Gill, C.A. and Dressler, E.A. 2005. Phenotypic ranges and relationships among carcass and meat palatability traits for fourteen cattle breeds, heritability and expected progeny differences for Warner-Bratzler shear force in three beef breeds. J Anim Sci, 83: 2461-2467.

Domingues, O.; Sanford, P.; Melo, J.M.; Maia, A.L. e Coelho, A.A. 1956. Preservação e seleção das raças nativas de gado do Nordeste. Seção de Fomento da Agricultura. Fortaleza. 28 pp.

Drake, D. e Chapman C.K. 2008. Using carcass data in the cowherd to make genetic improvement decisions. Cattle Producer's Library. www. ansci.colostate.edu/.../cattlemanslibrary/1042... (23/03/2011)

Egito, A.A.; Mariante, A.S. e Albuquerque, M.S.M. 2002. Programa brasileiro de conservação de recursos genéticos animais. Arch Zootec, 51: 39-52.

FAO. 2009. Climate change and the characterization, breeding and conservation of animal genetic resources. $40 \mathrm{pp}$.

Felício, P.E. 1998. Desdobramento da função qualidade da carne bovina. Higiene Alimentar, São Paulo, 12: 16-22.

Fitzhugh, H.A. and Strauss, M.S. 1992. Management of global animal genetic resources organizational and institutional structure. In: Hodges, J. The management of global animal genetic resources. Proceedings of a FAO Expert consultation. Rome. Italy. 309 pp.

Issa, É.C.; Jorge, W.; Egito, A.A. and Sereno, J.R.B. 2009. Cytogenetic analysis of the $y$ chromosome of native Brazilian bovine breed:

Archivos de zootecnia vol. 62, núm. 237, p. 19. 


\section{CARVALHO, FÉ DA SILVA, ALMEIDA, LIMANETO E BEFFA}

preliminary data. Arch Zootec, 58: 93-101. Junqueira, J.O. 1996. Qualidade das carcaças de bovinos joens, machos e fêmeas, cruzados Marchigiana vs. Nelore, terminadoss em confinamento. (Tese de mestrado). Faculdade de Zootecnia e engenharia de Alimentos. USP.

Mariante, A.S. e Cavalcante, N. 2006. Animais do descobrimento: raças domésticas da história do Brasil. Embrapa. Brasil. 274 pp.

McManus, C.; Prescott, E.; Paludo, G.R.; Bianchini, E.; Louvandini, H. and Mariante, A.S. 2008. Heat tolerance in naturalized Brazilian cattle breeds. Livest Sci, 120: 256-264.

Mazza, M.C.M.; Mazza, C.A.S.; Sereno, J.R.S.; Santos, S.A. and Moura, A.C. 1992. Phenotypical characterization of Pantaneiro cattle in Brazil. Arch Zootec, 41 (extra): 477-484.

Pereira, M.C.; Mercadante, M.E.Z.; Razook, A.G.; Figueiredo, L.A. and Albuquerque, L.G. 2008. Results of 23 years of selection for postweaning weight in a Caracu beef herd. $S$ Afr $J$ Anim Sci, 38: 136-144.

Primo, A.T. 1992. El ganado bovino ibérico en las Americas: 500 años despues. Arch Zootec, 41: 421-432.

Purchas, R.W. 1990. An assessment of role pH differences in determining the relative tenderness of meat from bulls and steers. Meat Sci, 27: 129-140.

SAS Institute. 1995. SAS software: user's guide, Cary. USA. 291 pp.

Shorthose, W.R. 1989. Dark-cutting in beef and sheep carcasses under different environment of Australia. In: Proceedings of an Australia workshop. Australian Meat and Livestock Research Development Corporation. Sydney. pp. 68-73.

Silva, N.V.; Silva, J.H.V.; Coelho, M.S.; Oliveira, E.R.A.; Araújo, J.A. e Amancio, A.L.L. 2008. Características de carcaça e carne ovina: uma abordagem das variáveis metodológicas e fatores de influência. Acta Vet Brasilica, 2: 103-110.

Trovo, J.B.F e Primo, A.T. 1984. Medidas morfológicas en bovinos Caracu. Em: Reunião Anual da Sociedade Brasileira de Zootecnia, 21. Anais... Belo Horizonte, SBZ.

Wheeler, T.L.; Cundiff, S.D.; Shackelford, S.D. and Koohmaraie, M. 2010. Characterization of biological types of cattle (Cycle VIII): Carcass, yield, and Longissimus palatability traits. J Anim Sci, 88: 3070-3083. 